\title{
Management of primary sclerosing cholangitis: Conventions and controversies
}

\author{
Natasha Chandok MD MPH${ }^{1}$, Gideon M Hirschfield MA MB BChir PhD²,3
}

\begin{abstract}
N Chandok, GM Hirschfield. Management of primary sclerosing cholangitis: Conventions and controversies. Can J Gastroenterol 2012;26(5):261-268.
\end{abstract}

Primary sclerosing cholangitis (PSC) is a chronic inflammatory cholangiopathy that results in fibrotic strictures and dilations of the intraand extrahepatic bile ducts. PSC is uncommon, occurs predominantly in males and has a strong association with inflammatory bowel disease. While the pathogenesis of PSC has not been fully elucidated, emerging evidence supports roles for the innate and adaptive immune systems, and genome-wide analyses have identified several genetic associations. Using the best available evidence, the present review summarizes the current understanding of the diagnosis, pathogenesis and management of PSC. Despite its rarity, there is an urgent need for collaborative research efforts to advance therapeutic options for PSC beyond liver transplantation.

Key Words: Diagnosis; Liver transplantation; Pathogenesis; Primary sclerosing cholangitis

Drimary sclerosing cholangitis (PSC) is a chronic inflammatory, large duct cholangiopathy that results in fibrotic strictures and dilations of the intra- and extrahepatic bile ducts. Unlike the more common small bile duct disease, primary biliary cirrhosis (PBC), PSC occurs predominantly in men and is commonly associated with inflammatory bowel disease (IBD), albeit with a subtle phenotypic difference (eg, rectal sparing, right-sided disease and backwash ileitis) from classic ulcerative colitis (UC) (1). Furthermore, it is clinically associated with cholangitis, biliary cirrhosis and progression to liver failure. An overarching fear for patients arises from an ominous relative increased risk of malignancy, both within the hepatobiliary tree and in the bowel, if associated with IBD.

The fundamental injury seen in PSC is not to hepatocytes, but rather to medium- and large-size bile ducts, with cholangiography demonstrating intra- and/or extrahepatic bile ducts with localized or multifocal strictures and intervening segments of normal or dilated ducts (2). Histologically, concentric periductal fibrosis ('onion skinning') is seen, with eventual progression to narrowing and obliteration of small bile ducts. The cholangiocytes demonstrate reactive features including expression of adhesion molecules, inflammatory and profibrogenetic cytokines, and receptors, as well as growth factors stimulating extracellular matrix production, accumulation and proliferation of periductal myofibroblasts. There is an increased prevalence of auotimmune disease in patients and their relatives and, while evidence to support genetic predisposition is emerging, nongenetic factors likely play a significant role in disease risk. The presence of a variety of secondary causes that can mimic the histological and radiological features of PSC (including autoimmune disease, ischemia, infection and toxins) suggests commonality in certain final pathways associated with biliary injury and that the primary insult may be heterogeneous (3).

The profound and deleterious fibro-obliterative process is clearly among the desired targets for new treatments. Presently, care is supportive and transplantation is offered as a surgical (life-saving) therapy

\author{
La prise en charge de la cholangite sclérosante \\ primitive : des conventions et des controverses
}

La cholangite sclérosante primitive (CSP) est une cholangiopathie inflammatoire chronique qui provoque des sténoses fibreuses et des dilatations des canaux cholédoques. La CSP, peu courante, se manifeste surtout chez les hommes et s'associe fortement aux maladies inflammatoires de l'intestin. La pathogenèse de la CSP n'est pas entièrement établie, mais des données probantes émergentes étayent le rôle des systèmes immunitaires inné et adaptatif, et des analyses génomiques ont permis d'établir plusieurs associations génétiques. Au moyen des meilleures données probantes, la présente analyse résume les connaissances actuelles sur le diagnostic, la pathogenèse et la prise en charge de la CSP. Malgré sa rareté, il est urgent de mener des recherches coopératives pour que les possibilités thérapeutiques ne se limitent plus à la greffe du foie.

\begin{abstract}
when the patient progresses to end stage $(4,5)$. The choleretic effects of ursodeoxycholic acid (UDCA) are associated with an improvement in liver biochemistry, but does not translate in to a credible survival benefit. Population-based studies from the Northern hemisphere, such as that by Lendvist et al (6) and others $(7,8)$ estimate the incidence and prevalence of PSC to be 1.2 per 100,000 and 16.2 per 100,000 , respectively, corresponding to earlier estimates from the Midwestern United States. Little in the way of consensus on the overall pathogenesis of PSC exists, and it remains the chronic liver disease with the greatest unmet clinical need. The present review addresses common concerns for practicing clinicians.
\end{abstract}

\section{PATHOGENESIS}

Overall, there is strong emerging evidence to demonstrate roles for both the innate and adaptive immune systems in disease pathogenesis (9). It is hoped that a better understanding of etiology will furnish clinicians and scientists with a greater opportunity to develop effective treatments. Notably, the pathways identified in PSC are distinct from those identified for PBC - a small bile duct lymphocytic cholangitis. Additionally, PSC is much less 'autoimmune' than PBC, at least genetically speaking. It is expected that future research will further define risk pathways using novel genetic approaches (eg, the Immunochip Project, fine mapping studies, exome sequencing) and increased sample size (eg, The International PSC Collaboration). Some of the emerging pathogenic themes are highlighted below.

\section{Lymphoyctes and PSC}

The lymphocytic infiltrate of PSC consists primarily of T cells with a predominance of $\mathrm{CD}^{+} \mathrm{T}$ cells with a $\mathrm{T}$ helper cell 1 phenotype (portal infiltrate) and $\mathrm{CD} 8^{+} \mathrm{T}$ cells (lobular infiltrate) (10). In addition to the $\alpha \beta$ T cells infiltrating the liver, there are increased proportions of $\gamma \delta^{+} \mathrm{T}$ cells in PSC, and the percentage of $\gamma \delta^{+} \mathrm{T}$ cells in peripheral

\footnotetext{
${ }^{1}$ Division of Gastroenterology, Western University, London; ${ }^{2}$ Department of Medicine, University of Toronto, Toronto, Ontario; ${ }^{3}$ Centre for Liver

Research, University of Birmingham, Birmingham, United Kingdom

Correspondence: Dr Natasha Chandok, Division of Gastroenterology, Western University, 339 Windermere Road, London, Ontario N6A 5A5.

Telephone 519-663-3002, fax 519-663-3858, e-mail chandok.n@gmail.com

Received for publication March 9, 2011. Accepted July 29, 2011
} 
blood of patients with PSC is greater than in healthy controls. The coexpression of the interleukin (IL)-2 receptor and CD45RO suggests they have an activated memory phenotype. Although the inflammatory infiltrate of patients with PSC is largely comprised of T cells, other cell types including natural killer (NK) cells, macrophages, B cells and biliary epithelial cells likely contribute to immunopathogenesis. The activation of the innate immune system as a primary event in PSC is also suggested, with disease potentially triggered by bacteria (or more likely their pathogen-associated molecular patterns) that enter the portal circulation through an inflamed permeable intestine. Immunoglobulin $\mathrm{G}$ directed against biliary epithelial cells has been found in the sera of some patients with PSC, and the binding of such antibodies to biliary epithelium appears to induce production of proinflammatory cytokines and upregulation of toll-like receptors.

Animal models of the disease that support 'infectious' triggers (eg, Helicobacter pylori) have been speculated by some to be relevant, with added biological corollaries to the sclerosing cholangitis seen in immunosuppressed patients with Cryptosporidial infection. As well, the cholangiographically similar autoimmune pancreatitis/sclerosing cholangitis has been associated with the presence of antibodies against a peptide with homology to an amino acid sequence of the plasminogen-binding protein of $\mathrm{H}$ pylori. The presence of perinuclear antineutrophil cytoplasmic antibodies (p-ANCA) is often noted in PSC (as well as other autoimmune liver diseases). Recently, it has been demonstrated that $\mathrm{p}$-ANCA in autoimmune liver disorders react with $\beta$-tubulin isotype 5 as autoantigen as well as with its bacterial precursor protein FtsZ, with the implication that the p-ANCA in autoimmune liver diseases probably reflects an abnormal immune response to intestinal microorganisms.

Mouse models of PSC remain limited (11), with the only promising model arising after targeted deletion of the murine biliary transporter MDR2. Secondary effects are recognized in this model, with disrupted tight junctions and basement membranes, bile acid leakage into portal tracts, induction of a portal inflammatory (CD11b, $\mathrm{CD}^{+}$) infiltrate and activation of proinflammatory (eg, tumour necrosis factor-alpha and IL-1 $\beta$ ) and profibrogenic cytokines (transforming growth factor- $\beta 1$ ). A sclerosing cholangiopathy is also encountered in adult patients with cystic fibrosis, raising the question for a role of cystic fibrosis transmembrane receptor (CFTR) variants in PSC $(12,13)$. In a subset of PSC patients with IBD, an increased prevalence of CFTR abnormalities was observed, and experimental induction of colitis in CFTR knockout mice resulted in the development of bile duct injury (14). While the association between CFTR mutations and human PSC is unclear, in a recent genome scan, the G-protein-coupled bile acid receptor 5 (also known as TGR5) was identified as a potential candidate gene (15). This molecule is involved in bile acid-induced fluid secretion in biliary epithelial cells, and can be found colocalized with CFTR and the apical sodiumdependent bile salt uptake transporter, suggesting a functional coupling of TGR5 to bile acid uptake and chloride secretion (16). Intriguingly, TGR5 has also been identified in cholangiocyte cilia, where it might conceivably couple biliary bile acid concentration and composition to ductular bile formation.

\section{Genes and PSC}

In 2010, the first genome-wide study in PSC (17) found, by far, the strongest genetic associations to be detected near human leukocyte antigen (HLA)-B at chromosome 6p21. Consistent with this first study, the latest PSC genome-wide association study (18) also found that the strongest associations with PSC were detected at single-nucleotide polymorphisms in the HLA complex at chromosome 6p21, peaking at rs3134792 in HLA-B $\left(\mathrm{P}=6.8 \times 10^{-49}\right)$. Inclusion of rs3134792 as a covariate demonstrated a complex residual association signal in the vicinity of the HLA class II region, suggesting the presence of multiple causative loci within the region. Previous studies of PSC investigated markers of innate immunity in close proximity to the HLA locus. A key set of NK receptors is the killer-cell immunoglobulin-like receptor (KIR), which bind HLA class I molecules, and the frequency of HLA-Bw4 and HLA-C2, ligands for the inhibitory KIRs 3DL1 and 2DL1, respectively, was significantly reduced in PSC patients compared with controls, suggesting an increase in NK cell activity by decreased inhibition (19). In addition to the decreased suppression of NK cell activity through HLA-KIR interactions, genetic evidence suggests a possible role for the direct activation of NK cells through the major histocompatibility complex class I chain-related (MIC) genes. MICA and MICB are encoded in the HLA region and their proteins activate NKG2D receptors on NK cells. A strong protective effect of the MICA*002 allele has been demonstrated, as has an increased allele frequency of MICA*008 (20). In contrast, a Norwegian study (19) found associations with the MICA5.1 and MICB24 alleles.

These two pivotal PSC genome-wide association scans have further identified five PSC risk loci outside the HLA complex, including $2 \mathrm{q} 13,2 \mathrm{q} 35,3 \mathrm{p} 21,10 \mathrm{p} 15$ and $13 \mathrm{q} 31$. Of these loci, $2 \mathrm{q} 35$ and $3 \mathrm{p} 21$ are also associated with UC. The causal genes for these regions still need to be definitively confirmed, but the locus on chromosome $2 \mathrm{q} 35$ harbours a compelling candidate gene (the bile acid receptor TGR5) as discussed above. BCL2L11 is an interesting candidate for the association at $2 \mathrm{q} 13$ because of its role in maintaining immunological tolerance. The BCL2L11 gene encodes the Bcl-2 interacting protein (Bim), which is crucial for maintaining immunological tolerance through induction of apoptosis of autoreactive T-lymphocytes, as well as the deletion of activated $\mathrm{T}$ cells after an immune response. Bcl2111 $1^{-/}$murine livers spontaneously demonstrate mononuclear cell infiltrates around intrahepatic bile ducts. IL2RA is of interest in PSC (10p15) because IL2 $\mathrm{ra}^{-1-}$ mice spontaneously develop intestinal and biliary inflammation. Significant association has also been confirmed for macrophage stimulating protein 1 (MST1) involved in regulation of the production of proinflammatory mediators. Interestingly, a PSCassociated amino acid change has been proposed to influence MST1receptor interaction, as well as risk of UC and Crohn's disease. The MST1 locus (3p21) likely represents an important overlapping susceptibility locus for PSC and IBD.

\section{Adhesion molecules and PSC}

A specific role for chemokines and their receptors has been highlighted for PSC, a disease often associated with colonic IBD (21-23). In this paradigm, long-lived memory lymphocytes arise as a result of colitis, and express homing receptors that direct subsequent migration not only to the gut but also the liver. Under normal circumstances, gut endothelium expresses a unique adhesion molecule known as mucosal addressin cell adhesion molecule-1 (MADCAM1), which is absent from other vascular beds, and a unique chemokine, CCL25, which is restricted to the small bowel. In PSC, hepatic inflammation leads to the upregulation of hepatic MADCAM1 and CCL25, and increased recruitment of mucosal T cells. Memory cells recirculate between the liver and gut but have the potential to become deleteriously activated by hepatic antigens. When characterized, one in five T cells infiltrating the liver in PSC can be shown to be interferon $\gamma$-producing $\alpha 4 \beta 7^{+}$CCR $9^{+}$memory/effector $\mathrm{T}$ cells, which are present at low frequencies in other liver disease. Such imprinting is dependent on gut dendritic cells because $\alpha 4 \beta 7$ and CCR9 are only colocalized on lymphocytes activated in the gut. Recent evidence indicates that gut-associated dendritic cells induce gut-homing receptors on $\mathrm{B}$ cells via a mechanism that depends on the vitamin A metabolite retinoic acid. Furthermore, the experimental inability of liver dendritic cells to similarly imprint gut tropism implies that the $\alpha 4 \beta 7^{+} \mathrm{CCR} 9^{+} \mathrm{T}$ cell infiltrate in PSC is primed in the gut. The functional relevance of $\alpha 4 \beta 7$ and CCR9 expression is supported by observations that both MADCAM1 and CCL25, which are absent from normal liver, are present on hepatic endothelium in liver diseases associated with colitis, and that $\alpha 4 \beta 7^{+}$CCR $9^{+}$ lymphocytes from the livers of patients with PSC bind MADCAM1 and respond to CCL25 in adhesion and migration assays. A murine model has now also provided in vivo evidence that the enterohepatic 
circulation of antigen-specific CD8 T cells can generate an autoimmune cholangitis.

\section{CLINICAL MANIFESTATIONS AND GENERAL DIAGNOSTIC CONSIDERATIONS}

PSC is a heterogeneous disease that manifests in a wide variety of ways across all age groups. While the classic patient is male with IBD presenting with symptoms in his third to fourth decade of life, increasingly, with ready access to liver biochemical screening and magnetic resonance imaging (MRI), patient presentation is spanning a wider spectrum. Asymptomatic early disease is more frequently identified while older patients are being seen with chronic disease into their seventh decade of life and beyond.

Clinical presentations range from an asymptomatic rise in alkaline phosphatase levels, to nonspecific fatigue and pruritus, to liver decompensation with profound jaundice and advanced features of portal hypertension. With early MRI, incidental diagnosis occurs, and patients with PSC need not have abnormal liver biochemistry. Additional complications associated with PSC include cholelithiasis, gallbladder carcinoma and colorectal cancer (CRC).

PSC is usually diagnosed on the basis of a chronic cholestatic derangement in liver enzyme levels (ie, alkaline phosphatase $\geq 1.5$ times upper limit of normal for at least six months) and fibrotic strictures of the intra- and/or extrahepatic biliary system. In practical terms, cholangiography by MRI is now, in essence, the primary diagnostic modality, with blood work and ultrasound used to exclude secondary etiologies that can mimic PSC. If cholangiography is normal, liver biopsy is indicated if no alternative explanation for a persistent cholestasis can be unearthed. A liver biopsy is not required to establish the diagnosis, but is of use when considering small-duct PSC, or an overlap with autoimmune hepatitis (AIH). Other laboratory abnormalities associated with PSC but nonessential for its diagnosis include p-ANCA, hypergammaglobulinemia and nonspecific antinuclear and smooth muscle autoantibodies.

Cardinal to securing the diagnosis of 'primary' sclerosing cholangitis is the exclusion of secondary causes of cholangitis including, but not limited to, biliary calculi, cholangiocarcinoma (CCA), biliary tract surgery, Caroli's disease, biliary toxin exposure, portal vein thrombosis, ischemic stricturing and alternative liver diseases that can cause biliary injury (eg, cholestatic drug-induced liver injury). It is particularly important for the clinician to also consider immunoglobulin $\mathrm{G}_{4}$-associated sclerosing cholangitis in the differential diagnosis of any patient presenting with biliary strictures given the characteristic high rate of steroid responsiveness of this entity, thereby averting long-term complications of stricturing biliary disease (24).

\section{Diagnostic imaging, invasive cholangiography and laboratory testing for CCA}

All patients require hepatobiliary imaging to establish the diagnosis, characterize the severity and/or complications of the disease and help exclude other conditions in the differential diagnosis. Ultrasound remains the first-line test of choice in the initial evaluation of patients with PSC due to its broad availability, low cost, excellent safety profile and high sensitivity for the detection of extrahepatic dominant strictures. Ultrasound is important to evaluate the patency of the portal vein and can also identify very early biliary wall thickening that may not be evident on MRI. This can help diagnostically in early stages of the disease (25). MRI with magnetic resonance cholangiopancreatography (MRCP) is, as previously stated, also commonly used in the assessment of PSC, but unlike ultrasound, MRI more reliably detects the presence of intrahepatic disease. Despite the superior safety of MRCP over endoscopic retrograde cholangiopancreatography (ERCP), MRCP has slightly inferior accuracy, in large part due to high interobserver variability (26). Practically, however, MRCP suffices as the diagnostic modality of choice, with there being little to be gained clinically for patients from the added risk of a diagnostic ERCP. Computed tomography cholangiography is an emerging but not widely available noninvasive modality for imaging the biliary tree. Preliminary
TABLE 1

Summary of the advantages and disadvantages of noninvasive imaging modalities in primary sclerosing cholangitis

\begin{tabular}{lll}
\hline $\begin{array}{l}\text { Imaging } \\
\text { modality }\end{array}$ & Advantages & Disadvantages \\
\hline Ultrasound & $\begin{array}{c}\text { Widely available and } \\
\text { inexpensive; no radiation; } \\
\text { good visualization of } \\
\text { extrahepatic biliary tree }\end{array}$ & $\begin{array}{c}\text { Poor sensitivity for } \\
\text { intrahepatic strictures; } \\
\text { poor specificity for } \\
\text { malignancy }\end{array}$ \\
$\begin{array}{lll}\text { Computed } \\
\text { tomography }\end{array}$ & $\begin{array}{l}\text { Widely available; specificity } \\
\text { for staging malignancy }\end{array}$ & $\begin{array}{c}\text { Radiation; computed } \\
\text { tomography }\end{array}$ \\
& superior to ultrasound & cholangiography protocols \\
Magnetic & Good visualization of intra- & High interobserver \\
resonance & and extrahepatic biliary & variability in reporting; \\
cholangiography & system; no ionizing & poor precision for ruling \\
& radiation; no risk of & out malignancy; inability \\
& cholangitis/pancreatitis & to be therapeutic \\
\hline
\end{tabular}

studies suggest it has a diagnostic accuracy comparable with MRCP in diagnosing PSC and dominant strictures; however, given the associated radiation and limited expertise in reporting, computed tomography cholangiography is used only in limited settings (27). A comparative summary of the advantages and disadvantages of noninvasive imaging modalities is presented in Table 1.

Although the disease course is variable and unpredictable, a systematic review of observational studies revealed that $10 \%$ to $20 \%$ of PSC patients have dominant strictures, and a significant proportion of these patients experience recurrent bacterial cholangitis (25). While the majority of dominant strictures are benign, up to $25 \%$ are malignant, but a definitive diagnosis of CCA is often challenging (25). Unfortunately, tumour markers, such as carbohydrate antigen (CA) 19-9, have suboptimal sensitivity and positive predictive value for the diagnosis of CCA (28). This remains a great challenge for patients and physicians, particularly given the need for high-quality ERCP studies. In the setting of a dominant stricture, it is critical to quickly identify the presence of malignancy because a timely diagnosis of early-stage CCA improves the likelihood of potentially life-saving surgical treatment. Regrettably, the radiological appearance of benign dominant strictures can appear identical to CCA. In such scenarios, tumour markers (ie, CA 19-9) and cytology should be pursued.

Cholangiography is the least invasive and most common means to obtain cytology. It is usually performed endoscopically or percutaneously; the former approach is preferred due to its superior safety profile. The associated risks of ERCP and percutaneous transhepatic cholagiography (PTC) include cholangitis, bleeding, pancreatitis and bowel perforation (29). Due to the potential for complications from ERCP, patients often undergo MRCP in lieu of, or before, invasive cholangiography despite the inferior diagnostic yield of MRCP. However, given that MRCP has a low sensitivity to diagnose CCA, clinicians should preferentially refer patients for ERCP if there is an absence of MRI expertise at their centre, and/or if there is imaging evidence or clinical suspicion for distal dominant stricturing. ERCP or PTC, rather than MRCP, is also more helpful to determine the feasibility of surgical resection, and is more logical if the patient is jaundiced in the absence of end-stage liver disease, given the chance for intervention.

Due to the risk of bacteremia with ERCP, available evidence supports the routine use of prophylactic antibiotics with Gram-negative coverage (30). There are no prospective data to determine whether dilation alone - versus a combination of dilation and stenting - is superior. Patients with dominant strictures amenable to ERCP frequently require several sessions to relieve biliary obstruction, but the treatment has a high success rate in improving symptoms and biochemistry in the majority of patients. PTC has similar efficacy to 


\begin{tabular}{|c|c|c|c|}
\hline Category & $\begin{array}{c}\text { Definitive } \\
\text { efficacy }\end{array}$ & $\begin{array}{c}\text { Level of } \\
\text { evidence* }\end{array}$ & Reference(s) \\
\hline \multicolumn{4}{|l|}{ Antibiotics } \\
\hline Metronidazole & No & $1 b$ & 71 \\
\hline Minocycline & No & $1 b$ & 72 \\
\hline \multicolumn{4}{|l|}{ Immunomodulatory } \\
\hline Corticosteroids & No & $1 b$ & $73-75$ \\
\hline Methotrexate & No & $1 \mathrm{~b}$ & $76-78$ \\
\hline Pencillamine & No & $1 b$ & 79 \\
\hline Azathioprine & No & $2 c$ & 80 \\
\hline Mycophenolate mofetil & No & $2 b$ & 81 \\
\hline Colchicine & No & $1 b, 3 b$ & 75,82 \\
\hline Etanercept & No & $2 b$ & 83 \\
\hline Infliximab & No & $1 \mathrm{~b}$ & 84 \\
\hline Cyclosporine & No & $1 \mathrm{~b}$ & 85 \\
\hline Tacrolimus & No & $2 b$ & 86 \\
\hline
\end{tabular}

*Adapted from the Centre for Evidence Based Medicine website <uww.cebm.net>

ERCP for distal stricturing, but is not preferred due to the higher rate of adverse events and the discomfort patients experience afterward with external catheters. Furthermore, PTC is technically difficult in the presence of intrahepatic disease.

Endoscopic ultrasound (EUS) is another minimally invasive, nonsurgical technique with outstanding ability to assess distal bile duct lesions and regional lymph nodes. EUS with final-needle aspiration has greater sensitivity and specificity for the diagnosis of distal CCA compared with ERCP and brush cytology. EUS has the added advantage of not contaminating the biliary tree (31).

Emerging invasive, nonsurgical technologies, such as cholangioscopy and intraductal ultrasound, hold promise but are not widely available, and more research is needed to assess their role in the arsenal of tests available for evaluation of the biliary system. In many patients with suspected biliary lesions, a staging laparotomy to ultimately determine resectability is required unless noninvasive imaging clearly shows distant metastases.

Because the sensitivity of cytology alone for the diagnosis of CCA is only $35 \%$ to $70 \%(32,33)$, combining cytology with molecular markers and novel genetic techniques is useful to improve the accuracy of diagnosis of CCA. The challenge, however, remains that these molecular techniques are not widely available and the practicalities of translating such ancillary testing to nontertiary centres is very unclear. It should also be noted that the presence of high-grade dysplasia alone on brush cytology has high sensitivity, specificity and positive predictive value for the diagnosis of CCA ( $73 \%, 95 \%$ and $85 \%$, respectively) (34).

Although CA 19-9 levels are frequently elevated in CCA, they are, unfortunately, also elevated in benign pancreaticobiliary conditions (particularly when patients are jaundiced) and other cancers such as adenocarcinoma of the pancreas. This severely limits the utility of measuring CA 19-9 and raises diagnostic problems when values are high but nothing is evident radiologically. The threshold value above which CA 19-9 is a useful diagnostic tool to rule in CCA depends on the clinical scenario in which it is measured. The sensitivity of CA 19-9 above $180 \mathrm{U} / \mathrm{mL}$ is only $67 \%$, but the specificity is better at $98 \%$ (35). In patients with cholangitis, a higher cut-off value of CA $19-9>300 \mathrm{U} / \mathrm{mL}$ to $400 \mathrm{U} / \mathrm{mL}$ is still associated with a poor sensitivity (41\%), but specificity remains high. While measuring CA 19-9 in patients with suspected CCA is reasonable, elevation of tumour marker levels alone is insufficient to establish the diagnosis. However, combining brush cytology with CA 19-9 increases the sensitivity to $88 \%$ (35).

Fluorescence in situ hybridization (FISH) and digital image analysis (DIA) are novel techniques to measure DNA proliferation and improve the diagnostic yield of cytology. FISH is a cytological method that uses DNA probes to identify a shortage or excess of chromosomes or chromosomal loci; these molecular derangements are common in CCA. Similarly, DIA quantifies the amount of cellular DNA by measuring intensity of nuclei stained with dye that binds to nuclear DNA. It is debatable as to what extent, if any, that FISH and DIA, in combination with cytology, improve the ability to diagnose CCA. The sensitivity and specificity for DNA aneuploidy in tumour detection is $43 \%$ and $96 \%$, respectively (36).

Given that both PSC and CCA are rare, patients with PSC and suspected CCA are likely best served at designated centres of excellence where clinical expertise and novel diagnostic testing are readily available. Practitioners should consider referring PSC patients with malignant appearing stricture(s) with moderate to severe atypia on brush cytology, with or without CA 19-9 above 100 U/L, to a hepatobiliary centre of excellence.

\section{MEDICAL MANAGEMENT CONSIDERATIONS}

Other than liver transplantation (LT), there are no proven effective treatments for PSC. An array of antimicrobials and immunomodulatory agents have been studied in the past two decades, with no robust demonstration of efficacy (Table 2).

\section{UDCA}

The role of UDCA in PSC is controversial, and requires further study before evidence-based recommendations can be finalized. As a therapeutic agent, UDCA is purported to have four mechanisms of action:

1. Protection of cholangiocytes against cytotoxic bile acids;

2. Promotion of hepatobiliary secretion;

3. Prevention of bile acid-induced apoptosis of hepatocytes; and

4. Induction of antioxidants.

Several PSC trials involving UDCA have repeatedly demonstrated an improvement in liver biochemistry, and smaller studies have even shown a tendency toward improved liver histology and cholangiogram appearances of strictures $(37,38)$. Furthermore, in a multicentre trial, Olsson et al (38) reported a tendency toward improved transplant-free survival in patients randomly assigned to $17 \mathrm{mg} / \mathrm{kg} /$ day to $23 \mathrm{mg} / \mathrm{kg} /$ day of UDCA versus placebo, but statistical significance was not achieved. Additionally, Pardi et al (39) provided evidence for a significant reduction in the risk of colonic dysplasia or CRC in patients with PSC and UC who received UDCA at $17 \mathrm{mg} / \mathrm{kg} /$ day to $23 \mathrm{mg} / \mathrm{kg} /$ day. The results of this latter study further propelled the routine use of UDCA for patients with PSC and IBD, despite the fact that this trial involving only 52 subjects was never replicated. It should be noted that highdose UDCA ( $28 \mathrm{mg} / \mathrm{kg} /$ day to $30 \mathrm{mg} / \mathrm{kg} /$ day) has been recently shown to increase the risk of colorectal neoplasia ( $\mathrm{HR} 4.44 ; \mathrm{P}=0.02)$ for unclear mechanisms (34).

In a randomized placebo-controlled trial, Lindor et al (40) showed that high-dose $(28 \mathrm{mg} / \mathrm{kg} /$ day to $30 \mathrm{mg} / \mathrm{kg} /$ day $)$ UDCA is not associated with improved transplant-free survival and, in fact, UDCA at this dosage may be harmful because subjects in the treatment arm of the study experienced higher frequencies of hepatic decompensation (40). Although the precise mechanism(s) of adverse events in patients with high-dose UDCA is unknown, it is possible that UDCA has a previously unrecognized therapeutic window (ie, at a very high dose, toxic levels of lithocholic acid may become relevant), and beyond a therapeutic dosage, UDCA may lead to prevention of apoptosis of stellate cells, which in turn could lead to accelerated fibrosis.

Based on the above results, high-dose UDCA at $28 \mathrm{mg} / \mathrm{kg} /$ day to $30 \mathrm{mg} / \mathrm{kg} /$ day has no role in the management of PSC, but UDCA at $17 \mathrm{mg} / \mathrm{kg} /$ day to $23 \mathrm{mg} / \mathrm{kg} /$ day may be therapeutic in selected patients pending further trials. It is conceivable that UDCA could modify disease during the early, inflammatory phase of biliary injury before fibrosis has ensued. This has left patients and clinicians confused. While it is clear that UDCA is not a panacea, and that high-dose UDCA is neither effective nor necessarily safe, the role for moderate 
dose (17 mg/kg/day to $23 \mathrm{mg} / \mathrm{kg} /$ day) UDCA is unclear. Multisociety guidelines are unable to concur on practical advice. As such, most clinicians now engage the patient in discussion and one practical strategy is to trial UDCA at $17 \mathrm{mg} / \mathrm{kg} / \mathrm{day}$ to $23 \mathrm{mg} / \mathrm{kg} / \mathrm{day}$, and continue it if there is a biochemical response. Although this 'art of practice' does not conform to 'evidence-based' medicine per se, it does resonate pragmatically with clinicians and patients, and there is no evidence for harm, other than arguably in monetary terms.

Other aspects of PSC management are less controversial and broadly involve either treatment of known complications of the disease or prevention of theoretical complications (Table 3). Modifiable complications include bacterial cholangitis, pruritus, malnutrition and hepatic osteodystrophy. Prevention of CRC and the role of LT will also be discussed.

\section{Bacterial cholangitis}

Cholangitis is a major cause of morbidity in the PSC population, but it should be borne in mind that it is unusual in early disease and that it is often precipitated by biliary intervention. Treatment of significant bacterial cholangitis begins with patient resuscitation and blood cultures, timely antimicrobial therapy targeting Gram-negative organisms, and subsequent attempt at relieving biliary obstruction with endoscopic or percutaneous cholangiography, if appropriate. However, some patients may experience mild episodes that they are able to manage at home with self-administration of oral ciprofloxacin. It is also appropriate to bear in mind that the classic presentation of pain, fever and leukocytosis need not be present in a patient with PSC, especially the more advanced and extensive their disease. Rarely, even biliary resection may need to be considered for stricturing disease not suitable for therapeutic cholangiography in the patient without advanced hepatic fibrosis (41). In the process of relieving stricturing disease, brushings and/or biopsies should be considered because CCA often presents as an innocent episode of cholangitis. Patients with recurrent cholangitis may benefit from prophylactic antibiotics to prevent septicemia, with the caveat that usually such patients ultimately progress toward LT.

\section{Pruritus}

Pruritus is a debilitating symptom that markedly diminishes a patient's quality of life. In its most classic form, pruritus is characterized by plantopalmar itch, sometimes relentless, and often worse at night. Concomitant depression and anxiety in the most severe of cases is inevitable. In addition to relief of biliary obstruction where appropriate, cholestyramine remains a reasonable first-line agent to prescribe. Additional agents that can be of benefit should be tried sequentially given the absence of an evidence-based algorithmic approach, with discontinuation of noneffective agents. Examples of antipruritic agents for which there is some evidence in cholestatic liver diseases include rifampin, sertraline, hydroxyzine and oral naltrexone. Unfortunately, the efficacy of these agents to improve pruritus is highly variable and a step-wise logical approach should be implemented (42). Severe pruritus refractory to oral agents may necessitate a trial of the Molecular Adsorbents Recirculating System (MARS). In a recent analysis of 20 patients who underwent MARS therapy for pruritus, $75 \%$ experienced clinical improvement with no severe adverse side effects (43). In the most severe cases, consideration for LT, irrespective of hepatic function, is warranted.

\section{Hepatic osteodystrophy}

Hepatic osteodystrophy is a prevalent complication of end-stage liver disease (ESLD), and particularly of chronic cholestatic liver diseases. Approximately $15 \%$ of patients with PSC have osteoporosis at the time of diagnosis, necessitating baseline and serial (ie, every two or three years) bone mineral density testing (44). In addition to appropriate supplementation with calcium and vitamin $\mathrm{D}$, patients with osteoporosis should receive bisphosphonate therapy to prevent further bone loss and fractures as per multisociety osteoporosis guidelines (4).
TABLE 3

Summary of the complications and management
considerations for primary sclerosing cholangitis

\begin{tabular}{lc}
\hline Complication & Management strategy considerations \\
\hline Cholangitis & Antibiotics, therapeutic cholangiography with dilation or \\
stent insertion & Antipruritic agents (see text). Relief of biliary obstruction \\
Pruritus & with therapeutic cholangiography \\
Hepatic & Calcium and vitamin D supplementation, bone density \\
osteodytrophy & screening, bisphosphosponate therapy for osteoporosis \\
Malnutrition & Protein and calorie supplementation. Screening and \\
& treatment of fat soluble vitamin deficiencies \\
Colorectal & Colonoscopy with segmental biopsies at diagnosis; annual \\
cancer & colonoscopy with segmental biopsies for surveillance in \\
surveillance & those with inflammatory bowel disease \\
Atypical & Obtain brush cytology +/- stricture biopsy, CA 19-9; \\
dominant & Review with expert pathologist, specimens with \\
strictures & moderate to severe atypia require careful follow-up \\
\hline
\end{tabular}

CA Carbohydrate antigen

Intravenous bisphosphonates may be an attractive option in subjects with esophageal varices in whom there is theoretical added concern over the risk of pill-induced upper gastrointestinal tract ulceration.

\section{Malnutrition}

Regardless of etiology, patients with ESLD have a propensity for sarcopenia due to their profound catabolic state. Patients with clinical features of malnutrition benefit from dietary assessment, and protein and calorie supplementation. In addition to protein and calorie malnutrition, patients with advanced cholestatic liver diseases are vulnerable to fat-soluble vitamin deficiencies. Once patients with PSC develop visible jaundice (total bilirubin level $>35 \mathrm{mmol} / \mathrm{L}$ ), they should receive annual surveillance and aggressive replacement of fat-soluble vitamins where indicated. Appropriate replacement of fat-soluble vitamins is crucial to prevent or treat complications such as osteopenia and osteoporosis, myalgia, night blindness, wound healing and muscle cramps, among others.

\section{Portal hypertension}

Patients with advanced PSC are prone to the garden-variety complications of portal hypertension, including variceal hemorrhage and ascites. Similar to the generic management of ESLD, patients with cirrhotic-stage PSC require routine endoscopy to survey for large varices. A platelet count of $<150 \times 10^{9} / \mathrm{L}$ in a patient with PSC is associated with an OR of 6.3 (95\% CI 2.6 to 15.8) to predict large varices (45). Becuase large varices can occur in the setting of normal range platelet counts in subjects with cholestatic liver diseases (46), any patient with features of portal hypertension, even in the absence of thrombocytopenia, should be considered for endoscopy to avert variceal hemorrhage through banding ligation or nonselective beta blockade.

\section{Surveillance for CRC}

As previously discussed, PSC is associated with both UC and Crohn's disease, but the former is a far stronger association. The PSC/UC overlap is considered a unique phenotype of IBD and, as previously stated, is associated with rectal sparing and backwash ileitis (47). In patients with PSC/UC, the RR of colorectal cancer is 10 -fold greater than in the general population and, hence, annual surveillance colonoscopy with segmental biopsies to assess for dysplasias is advised $(48,49)$. A meta-analysis of observational studies suggested that 5-aminosalicylic acid agents may be protective against colonic dysplasia and CRC (OR 0.51 [ $95 \%$ CI 0.38 to 0.69$]$ ) (50), and should thus be considered in PSC patients with UC. In the setting of colonic dysplasia or severe uncontrolled UC, a colectomy is warranted, although patients with advanced liver dysfunction and portal hypertension (Child-Pugh Class 
B or C disease) should be assessed for concomitant LT due to their high risk of hepatic failure with intra-abdominal surgery.

\section{Managing variant syndromes}

The diagnosis of variant syndromes associated with PSC, including overlap syndromes, small-duct PSC and sequential syndromes, is problematic due to the lack of standardized diagnostic criteria (51). While an approach to the diagnosis of variant syndromes is beyond the scope of the present review, the clinician should be mindful of a possible overlap with $\mathrm{AIH}$ in a PSC patient with a prominent hepatitic derangement in their liver enzyme profile not otherwise accounted for, and corroborating histopathological features of AIH. Published literature suggests that $5 \%$ to $30 \%$ of patients with PSC have concomitant AIH (52-54). Patients with PSC-AIH overlap frequently benefit from immunosuppression to prevent disease progression and liver failure. An overlap with immune-mediated hepatitis is much more common in children $(55,56)$. This condition has been called 'autoimmune sclerosing cholangitis' by pediatricians. It describes the situation by which it was identified: approximately $50 \%$ of children with classic $\mathrm{AIH}$ have cholangiographic features of PSC. Pediatricians are, therefore, likely to manage patients with immunosuppression and UDCA in this setting, although a robust evidence base for this is lacking. If clinicians see patients early in disease who are young, they should be more mindful to the possibility of a steroid responsive component of the disease but must be prudent before making the label.

Another consideration is that patients with PSC may also have a specific variant of the disease, small-duct PSC, diagnosed on the basis of a compatible clinical context and liver biopsy, and normal cholangiogram. Natural history studies suggest that small-duct PSC is merely an early stage of large-duct PSC, and the incidence of this entity is increasing over time $(8,57)$. Patients with small-duct PSC are usually asymptomatic and have minimal foreseeable risk of dire complications such as CCA $(57,58)$.

\section{LT}

The only proven curative treatment for PSC is LT. The decision for LT rests on several general considerations: quality of life of the potential recipient; longevity of life of the potential recipient; and organ availability and societal costs. Patients with advanced PSC have a particularly dismal quality of life due to the unique symptoms that afflict them, including pruritus and disproportionate fatigue; such symptoms are not directly captured by scoring systems used to evaluate LT candidates.

In most transplant programs in North America, qualified LT candidates are listed on the basis of the Model for End-stage Liver Disease (MELD) score. The MELD score, based on international normalized ratio, bilirubin and creatinine levels, provides a three-month mortality risk for potential recipients. Patients with the highest MELD score are given priority for LT in an effort to reduce the mortality rate among patients on the waiting list. While the MELD score may not encompass unique features specific to decompensated PSC, its benefit is that it is etiology independent and reproducible, and does not discriminate on the basis of patient demography or underlying disease. Further derivations of MELD, such as MELD-Na, are likely to better refine its overall utility in patients with advanced chronic liver disease.

Similar to any other LT candidate, the PSC patient with hepatocellular carcinoma (HCC) within Milan or equivalent criteria should receive MELD-exception points given the higher estimated threemonth mortality rate in such patients. While HCC is less common among PSC patients than other etiologies of ESLD, such as viral hepatitis, HCC occurs at a frequency of $1 \%$ to $2 \%$ per annum in patients with PSC.

More than HCC, CCA is the most feared malignancy among PSC patients, occurring at a frequency of $10 \%$ within 10 years of diagnosis of PSC, but most cases are diagnosed within the first two years $(59,60)$. While CCA is usually an absolute contraindication to LT, selected patients with early hilar CCA should be referred to a centre of excellence for experimental protocols involving LT in conjunction with neoadjuvant therapies, as modelled by an innovative program from the Mayo Clinic (USA).

Optimal timing of LT for PSC and other etiologies of ESLD is controversial. Presently, in programs with MELD-based allocation systems, patients are listed for deceased-donor hepatic allograft on the basis of the degree of hepatic dysfunction, with the sickest patients receiving priority. However, it is possible that this strategy may not achieve optimal clinical or cost effectiveness, and further studies are ongoing in the area of organ allocation to reduce the cost of LT and better address ethical principals such as justice and equality. Livedonor LT appears to have similar outcomes to deceased-donor transplantation in PSC and other etiologies of ESLD and, hence, may be an attractive option for patients with PSC who are often in the prime of their lives at the time of listing and, thus, stand to gain significant years of potential life.

There are unique surgical considerations in the operative and postoperative care of the PSC LT recipient. In the transplant operation, the default biliary anastomosis technique for PSC recipients is a Roux-Y choledochojejunostomy because the native common bile duct is often diseased, but there is evidence that duct-to-duct anastomosis can be safe and efficacious in selected patients with preserved native duct integrity (61). Postoperatively, recipients are reported to have a higher risk of hepatic artery thrombosis (62). Furthermore, patients with PSC, as well as other autoimmune diseases, are at greater risk for acute cellular rejection and chronic ductopenic rejection, and thus tend to require more generous immunosuppression in the early and late postoperative course $(63,64)$.

Post-transplant patient and graft survival rates for PSC and other autoimmune liver diseases are outstanding. For instance, in a study by the Mayo Clinic involving 150 consecutive patients with PSC (65), the one- and five-year patient and graft survival rates were $93.7 \%, 86.4 \%$; and $83.4 \%$ and $79 \%$, respectively. However, as part of the informed consent process for LT, patients with PSC should be aware that the recurrence rate of PSC after LT is approximately $20 \%$ after 10 years (66). To establish the diagnosis of recurrent PSC, causes of nonanastomotic biliary strictures must be excluded, including but not limited to, ischemic injury of the hepatic graft, graft derivation from donation after cardiac death, ABO incompatibility, hepatic artery thrombosis and cytomegalovirus infection. Risk factors for recurrent PSC include IBD, colectomy in patients with overlapping IBD, CCA before LT and history of acute cellular rejection $(65,66)$

With the improved survival of patients after LT regardless of underlying etiology, post-transplant malignancy is now a prevailing cause of late morbidity and mortality. Based on prospective data from the liver transplant database of the National Institute of Diabetes and Digestive and Kidney Diseases (67), the risk of skin and nonskin cancer after LT is, in fact, highest in recipients transplanted for PSC; these patients had a $19 \%$ incidence of skin cancer and $22 \%$ incidence of nonskin malignancy within 12 years of LT. LT recipients with a history of PSC are clearly at higher risk for CRC because of the added risks imposed by concomitant IBD and immunosuppression, but in addition to gastrointestinal malignancies, these patients experience more lymphoproliferative and skin malignancies than patients transplanted for other etiologies (67).

LT for hilar cholangiocarcinoma is an important consideration for the unfortunate patient with PSC-associated early hilar CCA in the context of a research protocol at a specialized CCA centre. Few tumours of hilar CCA are amenable to surgical cure, and the five-year survival rate for resection or transplantation alone is only approximately $30 \%(68,69)$. Due to the lack of acceptable treatment options, the Mayo Clinic pioneered a novel protocol using neoadjuvant therapy and LT in 1993. This specialized protocol combines the benefits of radiotherapy, chemosensitization and LT in select patients with unresectable hilar CCA fulfilling specific inclusion criteria (70). While most patients with hilar CCA would be outside of transplantation criteria at diagnosis due to the aggressive nature of the tumour, for 
patients with early diagnosis and tumour responsive to neoadjuvant treamtents, the one- and five-year patient survival rates are excellent (91\% and $73 \%$, respectively) (70).

\section{SUMMARY}

Our understanding of the pathogenesis of PSC is evolving rapidly, and the management of PSC is rife with conventions and controversies. To date, there are no proven medical treatments for PSC, and there is an urgent need for collaborative research in this area to provide efficacious therapeutic options to improve the associated high morbidity and mortality. Although high-dose UDCA has been refuted as a safe and effective agent for PSC, the role of low-dose UDCA requires clarification and remains disputed. The management of PSC is largely focused on the prevention and treatment of complications such as cholangitis, pruritus, malnutrition, hepatic osteodystrophy and, where overlap of IBD exists, prevention of CRC. Despite its rarity, and the fact that the majority of patients with PSC will never be affected, CCA is a grave concern in patients with PSC and, hence, a high index of suspicion should be maintained. LT remains the only effective and proven treatment for PSC, with five-year patient survival rates approximating $85 \%$ in the current era of transplantation. Select patients with limitedstage hilar CCA may benefit from LT with extensive neoadjuvant therapy, but such a specialized protocol remains experimental.

\section{REFERENCES}

1. Hirschfield GM, Heathcote EJ, Gershwin ME. Pathogenesis of cholestatic liver disease and therapeutic approaches. Gastroenterology 2010;139:1481-96.

2. Weismuller TJ, Wedemeyer J, Kubicka S, Strassburg CP, Manns MP. The challenges in primary sclerosing cholangitis aetiopathogenesis, autoimmunity, management and malignancy. J Hepatol 2008;48(Suppl 1):S38-57.

3. Webster GJ, Pereira SP, Chapman RW. Autoimmune pancreatitis/ $\mathrm{IgG}_{4}$-associated cholangitis and primary sclerosing cholangitis overlapping or separate diseases? J Hepatol 2009;51:398-402.

4. Chapman R, Fevery J, Kalloo A, et al. Diagnosis and management of primary sclerosing cholangitis. Hepatology 2010;51:660-78.

5. EASL Clinical Practice Guidelines: Management of cholestatic liver diseases. J Hepatol 2009;51:237-67.

6. Lindkvist B, Benito de Valle M, Gullberg B, Bjornsson E. Incidence and prevalence of primary sclerosing cholangitis in a defined adult population in Sweden. Hepatology 2010;52:571-7.

7. Bambha K, Kim WR, Talwalkar J, et al. Incidence, clinical spectrum, and outcomes of primary sclerosing cholangitis in a United States community. Gastroenterology 2003;125:1364-9.

8. Molodecky NA, Kareemi H, Parab R, et al. Incidence of primary sclerosing cholangitis: A systematic review and meta-analysis. Hepatology 2011;53:1590-9.

9. Bowlus CL. Cutting edge issues in primary sclerosing cholangitis. Clin Rev Allerg Immunol 2011;41:139-50.

10. Borchers AT, Shimoda S, Bowlus C, Keen CL, Gershwin ME. Lymphocyte recruitment and homing to the liver in primary biliary cirrhosis and primary sclerosing cholangitis. Semin Immunopathol 2009;31:309-22.

11. Pollheimer MJ, Trauner M, Fickert P. Will we ever model PSC? "It's hard to be a PSC model!" Clin Res Hepatol Gastroenterol 2011;35:792-804.

12. Henckaerts L, Jaspers M, Van Steenbergen W, et al. Cystic fibrosis transmembrane conductance regulator gene polymorphisms in patients with primary sclerosing cholangitis. J Hepatol 2009;50:150-7.

13. Pall $\mathrm{H}$, Zielenski J, Jonas MM, et al. Primary sclerosing cholangitis in childhood is associated with abnormalities in cystic fibrosismediated chloride channel function. J Pediatr 2007;151:255-9.

14. Blanco PG, Zaman MM, Junaidi O, et al. Induction of colitis in $\mathrm{cftr}^{-/}$mice results in bile duct injury. Am J Physiol Gastrointest Liver Physiol 2004;287:G491-6.
15. Hov JR, Keitel V, Schrumpf E, Haussinger D, Karlsen TH. TGR5 Sequence variation in primary sclerosing cholangitis. Digestive Dis 2011;29:78-84.

16. Keitel V, Ullmer C, Haussinger D. The membrane-bound bile acid receptor TGR5 (Gpbar-1) is localized in the primary cilium of cholangiocytes. Biological Chem 2010;391:785-9.

17. Karlsen TH, Franke A, Melum E, et al. Genome-wide association analysis in primary sclerosing cholangitis. Gastroenterology 2010;138:1102-11

18. Melum E, Franke A, Schramm C, et al. Genome-wide association analysis in primary sclerosing cholangitis identifies two non-HLA susceptibility loci. Nature Genet 2011;43:17-9.

19. Karlsen TH, Boberg KM, Olsson M, et al. Particular genetic variants of ligands for natural killer cell receptors may contribute to the HLA associated risk of primary sclerosing cholangitis. J Hepatol 2007;46:899-906.

20. Norris S, Kondeatis E, Collins R, et al. Mapping MHC-encoded susceptibility and resistance in primary sclerosing cholangitis: The role of MICA polymorphism. Gastroenterology 2001;120:1475-82.

21. Adams DH. Mucosal lymphocytes in the pathogenesis of the hepatic complications of inflammatory bowel disease. Clin Med 2008;8:28-9.

22. Eksteen B, Mora JR, Haughton EL, et al. Gut homing receptors on CD8 $T$ cells are retinoic acid dependent and not maintained by liver dendritic or stellate cells. Gastroenterology 2009;137:320-9.

23. Oo YH, Shetty S, Adams DH. The role of chemokines in the recruitment of lymphocytes to the liver. Digestive Dis 2010;28:31-44.

24. Hirano $\mathrm{K}$, Tada M, Isayama $\mathrm{H}$, et al. Long-term prognosis of autoimmune pancreatitis with and without corticosteroid treatment. Gut 2007;56:1719-24.

25. Aljiffry M, Renfrew PD, Walsh MJ, Laryea M, Molinari M. Analytical review of diagnosis and treatment strategies for dominant bile duct strictures in patients with primary sclerosing cholangitis. HPB 2011;13:79-90.

26. Moff SL, Kamel IR, Eustace J, et al. Diagnosis of primary sclerosing cholangitis: A blinded comparative study using magnetic resonance cholangiography and endoscopic retrograde cholangiography. Gastrointest Endosc 2006;64:219-23.

27. Eracleous E, Genagritis M, Papanikolaou N, et al. Complementary role of helical CT cholangiography to MR cholangiography in the evaluation of biliary function and kinetics. Eur Radiol 2005;15:2130-9.

28. Levy MJ, Baron TH, Clayton AC, et al. Prospective evaluation of advanced molecular markers and imaging techniques in patients with indeterminate bile duct strictures. Am J Gastroenterol 2008;103:1263-73.

29. Harbin WP, Mueller PR, Ferrucci JT Jr. Transhepatic cholangiography: Complicatons and use patterns of the fine-needle technique: A multi-institutional survey. Radiology 1980;135:15-22.

30. Brand M, Bizos D, O'Farrell P Jr. Antibiotic prophylaxis for patients undergoing elective endoscopic retrograde cholangiopancreatography. Cochrane Database Syst Rev 2010;(10):CD007345.

31. Abu-Hamda EM, Baron TH. Endoscopic management of cholangiocarcinoma. Semin Liv Dis 2004;24:165-75.

32. Desa LA, Akosa AB, Lazzara S, Domizio P, Krausz T, Benjamin IS. Cytodiagnosis in the management of extrahepatic biliary stricture. Gut 1991;32:1188-91.

33. Mansfield JC, Griffin SM, Wadehra V, Matthewson K. A prospective evaluation of cytology from biliary strictures. Gut 1997;40:671-7.

34. Eaton JE, Silveira MG, Pardi DS, et al. High-dose ursodeoxycholic acid is associated with the development of colorectal neoplasia in patients with ulcerative colitis and primary sclerosing cholangitis. Am J Gastroenterol 2011;106:1638-45.

35. Siqueira E, Schoen RE, Silverman W, et al. Detecting cholangiocarcinoma in patients with primary sclerosing cholangitis. Gastrointest Endosc 2002;56:40-7.

36. Lindberg B, Enochsson L, Tribukait B, Arnelo U, Bergquist A. Diagnostic and prognostic implications of DNA ploidy and S-phase evaluation in the assessment of malignancy in biliary strictures. Endoscopy 2006;38:561-5.

37. Mitchell SA, Bansi DS, Hunt N, Von Bergmann K, Fleming KA, Chapman RW. A preliminary trial of high-dose ursodeoxycholic acid in primary sclerosing cholangitis. Gastroenterology 2001;121:900-7. 
38. Olsson R, Boberg KM, de Muckadell OS, et al. High-dose ursodeoxycholic acid in primary sclerosing cholangitis: A 5-year multicenter, randomized, controlled study. Gastroenterology 2005;129:1464-72.

39. Pardi DS, Loftus EV, Jr., Kremers WK, Keach J, Lindor KD. Ursodeoxycholic acid as a chemopreventive agent in patients with ulcerative colitis and primary sclerosing cholangitis. Gastroenterology 2003;124:889-93.

40. Lindor KD, Kowdley KV, Luketic VA, et al. High-dose ursodeoxycholic acid for the treatment of primary sclerosing cholangitis. Hepatology 2009;50:808-14.

41. Tsai S, Pawlik TM. Primary sclerosing cholangitis: The role of extrahepatic biliary resection. Advances Surg 2009;43:175-88.

42. Kremer AE, Oude Elferink RP, Beuers U. Pathophysiology and current management of pruritus in liver disease. Gastroenterol Clin Biol 2011, January (Epub ahead of print).

43. Pares A, Herrera M, Aviles J, Sanz M, Mas A. Treatment of resistant pruritus from cholestasis with albumin dialysis: Combined analysis of patients from three centers. J Hepatol 2010;53:307-12.

44. Angulo P, Grandison GA, Fong DG, et al. Bone disease in patients with primary sclerosing cholangitis. Gastroenterology 2011;140:180-8.

45. Zein CO, Lindor KD, Angulo P. Prevalence and predictors of esophageal varices in patients with primary sclerosing cholangitis. Hepatology 2004;39:204-10.

46. Bressler B, Pinto R, El-Ashry D, Heathcote EJ. Which patients with primary biliary cirrhosis or primary sclerosing cholangitis should undergo endoscopic screening for oesophageal varices detection? Gut 2005;54:407-10.

47. Loftus EV Jr, Harewood GC, Loftus CG, et al. PSC-IBD: A unique form of inflammatory bowel disease associated with primary sclerosing cholangitis. Gut 2005;54:91-6.

48. Leddin D, Hunt R, Champion M, et al. Canadian Association of Gastroenterology and the Canadian Digestive Health Foundation: Guidelines on colon cancer screening. Can J Gastroenterol 2004;18:93-9.

49. Loftus EV Jr, Sandborn WJ, Tremaine WJ, et al. Risk of colorectal neoplasia in patients with primary sclerosing cholangitis. Gastroenterology 1996;110:432-40.

50. Velayos FS, Terdiman JP, Walsh JM. Effect of 5-aminosalicylate use on colorectal cancer and dysplasia risk: A systematic review and meta-analysis of observational studies. Am J Gastroenterol 2005; $100: 1345-53$.

51. Boberg KM, Chapman RW, Hirschfield GM, Lohse AW, Manns MP, Schrumpf E. Overlap syndromes: The International Autoimmune Hepatitis Group (IAIHG) position statement on a controversial issue. J Hepatol 2011;54:374-85.

52. Gheorghe L, Iacob S, Gheorghe C, et al. Frequency and predictive factors for overlap syndrome between autoimmune hepatitis and primary cholestatic liver disease. Eur J Gastroenterol Hepatol 2004;16:585-92.

53. Muratori L, Cassani F, Pappas G, et al. The hepatitic/cholestatic "overlap" syndrome: An Italian experience. Autoimmunity 2002;35:565-8.

54. Kaya M, Angulo P, Lindor KD. Overlap of autoimmune hepatitis and primary sclerosing cholangitis: An evaluation of a modified scoring system. J Hepatol 2000;33:537-42.

55. Gregorio GV, Portmann B, Karani J, et al. Autoimmune hepatitis/ sclerosing cholangitis overlap syndrome in childhood: A 16-year prospective study. Hepatology 2001;33:544-53.

56. Mieli-Vergani G, Vergani D. Unique features of primary sclerosing cholangitis in children. Curr Opin Gastroenterol 2010;26:265-8.

57. Bjornsson E, Olsson R, Bergquist A, et al. The natural history of small-duct primary sclerosing cholangitis. Gastroenterology 2008;134:975-80.

58. Singal AK, Stanca CM, Clark V, et al. Natural history of small duct primary sclerosing cholangitis: A case series with review of the literature. Hepatol Int 2011;5:808-13.

59. Burak K, Angulo P, Pasha TM, Egan K, Petz J, Lindor KD. Incidence and risk factors for cholangiocarcinoma in primary sclerosing cholangitis. Am J Gastroenterol 2004;99:523-6.

60. Boberg KM, Bergquist A, Mitchell S, et al. Cholangiocarcinoma in primary sclerosing cholangitis: Risk factors and clinical presentation. Scand J Gastroenterol 2002;37:1205-11.

61. Heffron TG, Smallwood GA, Ramcharan T, et al. Duct-to-duct biliary anastomosis for patients with sclerosing cholangitis undergoing liver transplantation. Transplant Proc 2003;35:3006-7.
62. Wiesner RH. Liver transplantation for primary sclerosing cholangitis: Timing, outcome, impact of inflammatory bowel disease and recurrence of disease. Best Pract Res Clin Gastroenterol 2001; 15:667-80.

63. Florman S, Schiano T, Kim L, et al. The incidence and significance of late acute cellular rejection ( $>1000$ days) after liver transplantation. Clin Transplant 2004;18:152-5.

64. Graziadei IW, Wiesner RH, Marotta PJ, et al. Long-term results of patients undergoing liver transplantation for primary sclerosing cholangitis. Hepatology 1999;30:1121-7.

65. Graziadei IW, Wiesner RH, Batts KP, et al. Recurrence of primary sclerosing cholangitis following liver transplantation. Hepatology 1999;29:1050-6.

66. Campsen J, Zimmerman MA, Trotter JF, et al. Clinically recurrent primary sclerosing cholangitis following liver transplantation: A time course. Liver Transplant 2008;14:181-5.

67. Watt KD, Pedersen RA, Kremers WK, Heimbach JK, Sanchez W, Gores GJ. Long-term probability of and mortality from de novo malignancy after liver transplantation. Gastroenterology 2009;137:2010-7.

68. Meyer CG, Penn I, James L. Liver transplantation for cholangiocarcinoma: Results in 207 patients. Transplantation 2000;69:1633-7.

69. Robles R, Figueras J, Turrion VS, et al. Liver transplantation for hilar cholangiocarcinoma: Spanish experience. Transplant Proc 2003;35:1821-2.

70. Rosen CB, Heimbach JK, Gores GJ. Liver transplantation for cholangiocarcinoma. Transplant Int 2010;23:692-7.

71. Farkkila M, Karvonen AL, Nurmi H, et al. Metronidazole and ursodeoxycholic acid for primary sclerosing cholangitis: A randomized placebo-controlled trial. Hepatology 2004;40:1379-86.

72. Silveira MG, Torok NJ, Gossard AA, et al. Minocycline in the treatment of patients with primary sclerosing cholangitis: Results of a pilot study. Am J Gastroenterol 2009;104:83-8.

73. Angulo P, Batts KP, Jorgensen RA, LaRusso NA, Lindor KD. Oral budesonide in the treatment of primary sclerosing cholangitis. Am J Gastroenterol 2000;95:2333-7.

74. Kochhar R, Goenka MK, Das K, et al. Primary sclerosing cholangitis: An experience from India. J Gastroenterol Hepatol 1996;11:429-33.

75. Lindor KD, Wiesner RH, Colwell LJ, Steiner B, Beaver S, LaRusso NF. The combination of prednisone and colchicine in patients with primary sclerosing cholangitis. Am J Gastroenterol 1991;86:57-61.

76. Kaplan MM, Arora S, Pincus SH. Primary sclerosing cholangitis and low-dose oral pulse methotrexate therapy. Clinical and histologic response. Ann Intern Med 1987;106:231-5.

77. Knox TA, Kaplan MM. Treatment of primary sclerosing cholangitis with oral methotrexate. Am J Gastroenterol 1991;86:546-52.

78. Knox TA, Kaplan MM. A double-blind controlled trial of oral-pulse methotrexate therapy in the treatment of primary sclerosing cholangitis. Gastroenterology 1994;106:494-9.

79. Gross JB, Jr., Ludwig J, Wiesner RH, McCall JT, LaRusso NF. Abnormalities in tests of copper metabolism in primary sclerosing cholangitis. Gastroenterology 1985;89:272-8.

80. Kaplan MM. Medical approaches to primary sclerosing cholangitis. Semin Liver Dis 1991;11:56-63.

81. Talwalkar JA, Angulo P, Keach JC, Petz JL, Jorgensen RA, Lindor KD. Mycophenolate mofetil for the treatment of primary sclerosing cholangitis. Am J Gastroenterol 2005;100:308-12.

82. Olsson R, Broome U, Danielsson A, et al. Colchicine treatment of primary sclerosing cholangitis. Gastroenterology 1995;108:1199-203.

83. Epstein MP, Kaplan MM. A pilot study of etanercept in the treatment of primary sclerosing cholangitis. Dig Dis Sci 2004;49:1-4

84. Hommes DW Erkelens W, Ponsioen C, et al. A double-blind, placebo-controlled, randomized study of infliximab in primary sclerosing cholangitis. J Clin Gastroenterol 2008;42:522-6.

85. Wiesner RH, Steiner B, LaRusso NA. A controlled clinical trial evaluating cyclosporine in the treatment of primary sclerosing cholangitis. Hepatology 1991;14:63A (Abst).

86. Van Thiel DH, Carroll P, Abu-Elmagd K, et al. Tacrolimus (FK 506), a treatment for primary sclerosing cholangitis: Results of an open-label preliminary trial. Am J Gastroenterol 1995;90:455-9. 


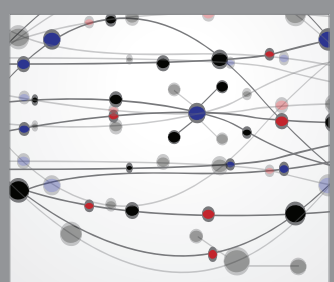

The Scientific World Journal
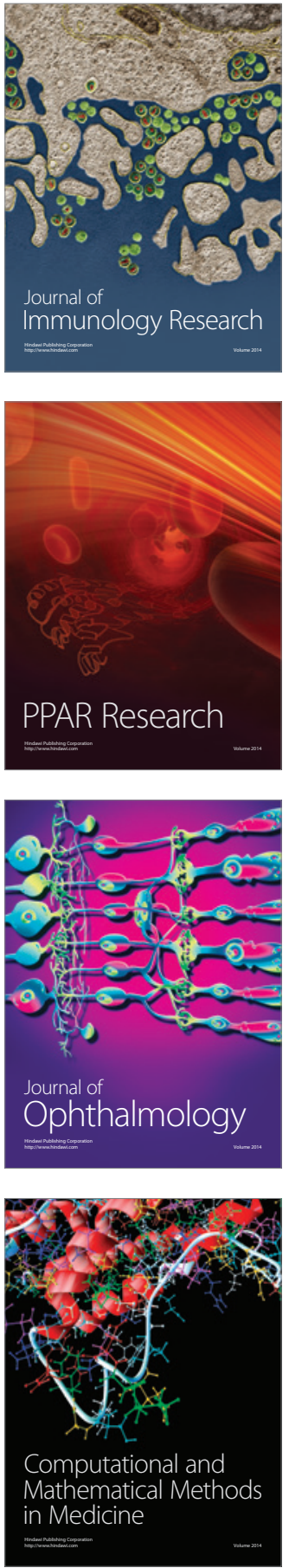

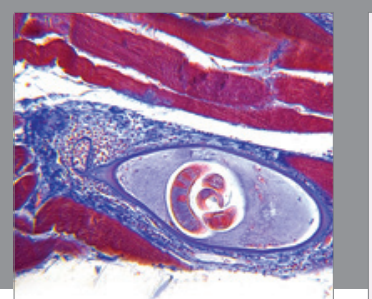

Gastroenterology Research and Practice

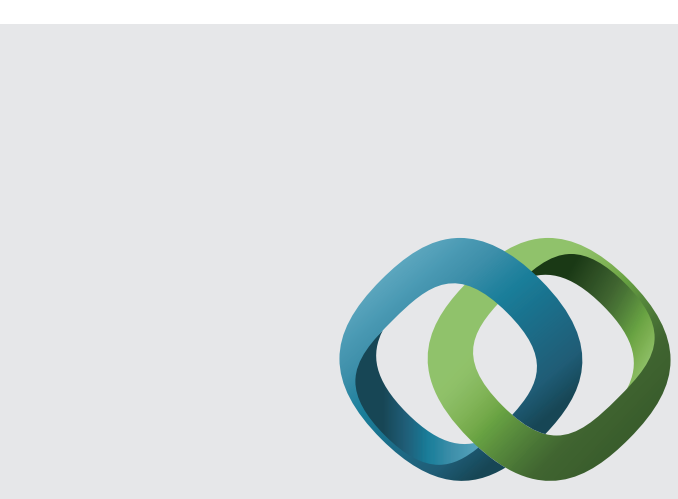

\section{Hindawi}

Submit your manuscripts at

http://www.hindawi.com
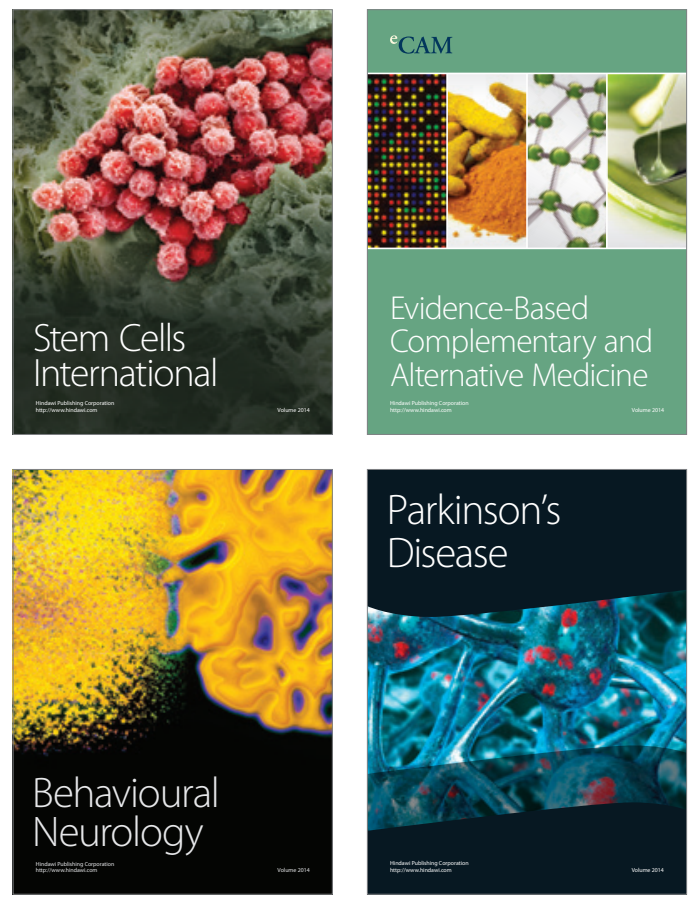
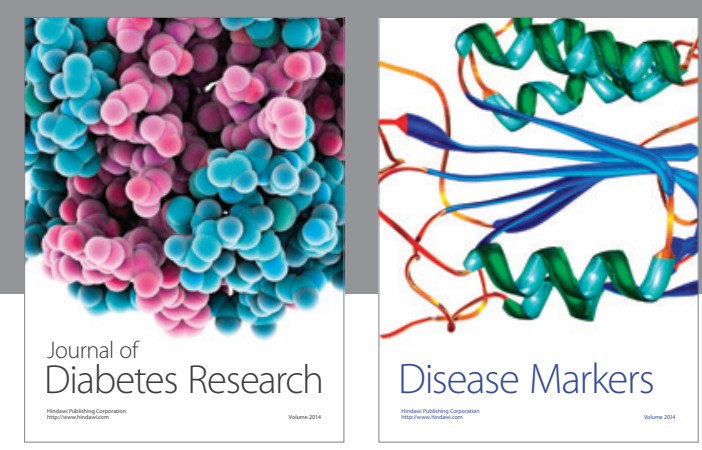

Disease Markers
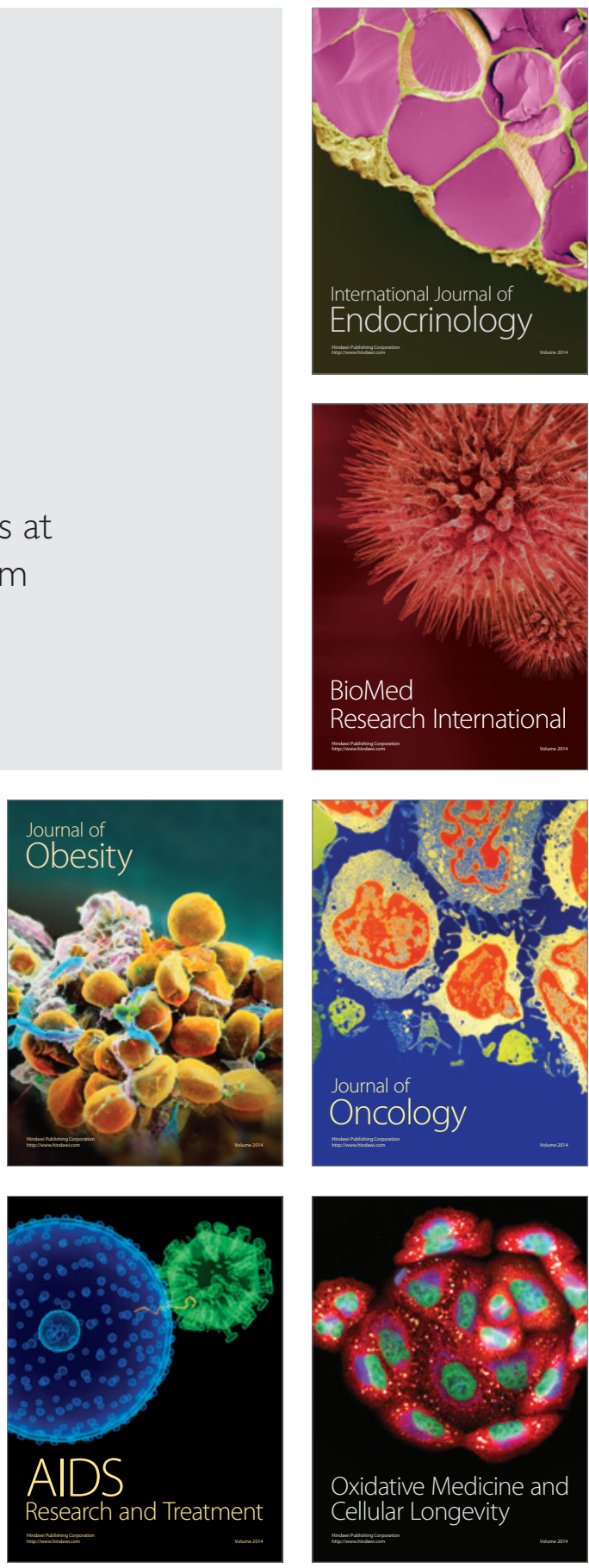